\title{
On the Middle Coefficient of a Cyclotomic Polynomial
}

\author{
Gregory P. Dresden
}

The cyclotomic polynomials $\Phi_{n}$ for $n=1,2,3, \ldots$ (familiar to every student of algebra) are the minimal polynomials for the primitive $n$th roots of unity:

$$
\Phi_{n}(x)=\prod_{(k, n)=1}\left(x-e^{2 \pi i k / n}\right)
$$

Clearly $\Phi_{n}$ has degree $\phi(n)$, where $\phi$ signifies Euler's totient function. These monic polynomials can be defined recursively as $\Phi_{1}(x)=x-1$ and $\prod_{i \mid n} \Phi_{i}(x)=x^{n}-1$ for $n>1$. The first few are easily calculated to be $x-1, x+1, x^{2}+x+1, x^{2}+1, \ldots$ For these and other basic facts, see an algebra text such as [5].

While it might appear that the coefficients of the cyclotomic polynomials are always \pm 1 , the presence of $2 x^{7}$ in $\Phi_{105}(x)$ shows that this is not invariably the case (and indeed is a good counterexample for those students who insist that the "law of small numbers" is universally valid; see [4] for further discussion). Naturally, much work has been done on the values of the coefficients of $\Phi_{n}(x)$. One amazing fact worthy of mention is that every integer appears as a coefficient in some cyclotomic polynomial (see [1], [8]).

In this article, we provide a short and elementary proof of the following result:

Theorem 1. For $n \geq 3$ the middle coefficient of $\Phi_{n}(x)$ is either zero (when $n$ is a power of 2) or an odd integer.

A similar result can be found in [6], where Lam and Leung directly calculate the middle coefficient of $\Phi_{p q}(x)$ for distinct primes $p$ and $q$ and show it to be \pm 1 . This had been done earlier by Beiter [2] for the case of distinct odd primes. Both papers rely on the partition of $\phi(p q) / 2$ into $r p+s q$. In contrast, our proof uses only some very basic facts about minimal polynomials. We also point out that for $n \neq p q$ the polynomial $\Phi_{n}(x)$ could indeed have a middle coefficient different from 1 or -1 . The first such occurence is at $n=385$ 
(giving a middle coefficient of -3 ), after which one sees 5 at $n=4785$, followed by -7 at $n=7735$, and 19 at $n=11305$. All these values of $n$ are square-free products of small odd primes, which is alluded to in [8].

Before proceeding with the proof of Theorem 1, we do a bit of preliminary work. The first lemma establishes a useful fact about $\Phi_{n}(x)$.

Lemma 1. If $n \geq 3$ and is odd, then $\Phi_{n}(-1)=1$.

Proof. For $n \geq 3$,

$$
\prod_{i \mid n, i>1} \Phi_{i}(x)=\frac{x^{n}-1}{x-1}
$$

so (since $n$ odd)

$$
\prod_{i \mid n, i>1} \Phi_{i}(-1)=\frac{(-1)^{n}-1}{(-1)-1}=1 .
$$

Also, $\Phi_{3}(-1)=1$. By a simple induction argument we conclude that $\Phi_{n}(-1)=1$ whenever $n \geq 3$ and is odd.

Next we review some basic information. We use $\zeta_{n}$ to signify a primitive $n$th root of unity (that is, $\zeta_{n}=e^{2 \pi i k / n}$ for some $k$ relatively prime to $n$ ), and $f_{n}(x)$ to denote the minimal polynomial of $\zeta_{n}+\zeta_{n}^{-1}$ (recall that the minimal polynomial of an algebraic complex number $\alpha$ is the monic polynomial $p(x)$ in $\mathbb{Q}[x]$ of smallest degree such that $p(\alpha)=0)$. It is not hard to show using elementary methods (see [7]) that $f_{n}$ has integer coefficients and that when $n \geq 3$ the degree of $f_{n}$ is half that of $\Phi_{n}(x)$. In fact,

$$
\Phi_{n}(x)=f_{n}\left(x+x^{-1}\right) \cdot x^{\phi(n) / 2} \quad(n \geq 3)
$$

because (after simplifying the right-hand side) the polynomials on both sides of (1) are monic, are of degree $\phi(n)$, and have $\zeta_{n}$ as a root. The first few such polynomials $f_{n}$ (for $n \geq 3$ ) are easy to derive from (1) and read as follows:

$$
\begin{array}{lll}
f_{3}(x)=x+1, & f_{5}(x)=x^{2}+x-1, & f_{7}(x)=x^{3}+x^{2}-2 x-1, \\
f_{4}(x)=x, & f_{6}(x)=x-1, & f_{8}(x)=x^{2}-2 .
\end{array}
$$


From this, we see that the constant term in $f_{n}$ is not always \pm 1 (equivalently, $\zeta_{n}+\zeta_{n}^{-1}$ is not necessarily an algebraic unit, meaning a unit in the ring of algebraic integers). However, by doing a careful comparison of the $f_{n}$ with the Chebyshev polynomials, Carlitz and Thomas [3] showed that when $n \geq 3$ and $n$ is not divisible by 4 , the constant term in $f_{n}(x)$ is either 1 or -1 . For the sake of completeness, we provide a nonelementary, but much shorter, proof of this fact.

Lemma 2. If $n \geq 3$ and $n \not \equiv 0(\bmod 4)$, then $\zeta_{n}+\zeta_{n}^{-1}$ is an algebraic unit.

Proof. Let $m=n$ for $n$ odd and $m=n / 2$ for $n$ even. Note that $m$ is itself odd and $m \geq 3$. Note as well that $\zeta_{n}{ }^{2}$ is a primitive $m$ th root of unity (and thus a root of $\Phi_{m}(x)$ ). Then $\zeta_{n}{ }^{2}+1$ is a root of $\Phi_{m}(x-1)$, which is a monic polynomial with constant term $\Phi_{m}(-1)=1$ (by Lemma 1). It follows that $\zeta_{n}{ }^{2}+1$ is an algebraic unit, as is $\zeta_{n}$. Thus, $\zeta_{n}+\zeta_{n}^{-1}=\left(\zeta_{n}{ }^{2}+1\right) / \zeta_{n}$ is likewise an algebraic unit.

We are now ready to bring everything together.

Proof of Theorem 1. If $n=2^{k}$, then $\Phi_{n}(x)=x^{2^{k-1}}+1$, a polynomial with zero as its middle coefficient. We proceed assuming that $n$ is not a power of 2 .

Note that if $\zeta$ is a primitive $4 k$ th root of unity, then $\zeta^{2}$ is a primitive $2 k$ th root of unity. Since $\phi(4 k)=2 \phi(2 k)$, we know that $\Phi_{4 k}(x)=\Phi_{2 k}\left(x^{2}\right)$. Since the middle coefficient of $\Phi_{2 k}\left(x^{2}\right)$ is the same as that of $\Phi_{2 k}(x)$, we can further assume without loss of generality that 4 does not divide $n$.

Now letting $f_{n}(x)$ be the minimal polynomial of $\zeta_{n}+\zeta_{n}^{-1}$, we know from Lemma 2 that $f_{n}$ has constant coefficient \pm 1 . Thus, we can write $f_{n}(x)=x^{k}+a_{k-1} x^{k-1}+\cdots+a_{1} x \pm 1$ (for $k=\phi(n) / 2$ ), and so from equation (1) we obtain

$$
\Phi_{n}(x)=\left[\left(x+x^{-1}\right)^{k}+a_{k-1}\left(x+x^{-1}\right)^{k-1}+\cdots \pm 1\right] \cdot x^{k} .
$$

The middle coefficient of $\Phi_{n}(x)$ is the coefficient of the $x^{k}$ term in (2) (recall, $\left.k=\phi(n) / 2\right)$. This number is simply the sum of the constant terms appearing in each expression $a_{i}\left(x+x^{-1}\right)^{i}$ in (2), plus the final \pm 1 . The constant term in $a_{i}\left(x+x^{-1}\right)^{i}$ is either zero (for $i$ odd) or $a_{i}\left(\begin{array}{c}i \\ i / 2\end{array}\right)$ 
(for $i$ even). As a result, the middle coefficient of $\Phi_{n}(x)$ is

$$
\sum_{i=2 j} a_{i}\left(\begin{array}{c}
i \\
i / 2
\end{array}\right) \pm 1=\sum_{j} a_{2 j}\left(\begin{array}{c}
2 j \\
j
\end{array}\right) \pm 1 .
$$

By a familiar identity,

$$
\left(\begin{array}{c}
2 j \\
j
\end{array}\right)=\left(\begin{array}{c}
2 j-1 \\
j-1
\end{array}\right)+\left(\begin{array}{c}
2 j-1 \\
j
\end{array}\right)=2\left(\begin{array}{c}
2 j-1 \\
j
\end{array}\right) .
$$

Thus the middle coefficient of $\Phi_{n}(x)$ is odd when $n$ is not a power of 2 .

\section{References}

[1] S. D. Adhikari, S. A. Katre, and D. Thakur, eds., Cyclotomic Fields and Related Topics, Bhaskaracharya Pratishthana, Pune, 2000.

[2] M. Beiter, The midterm coefficient of the cyclotomic polynomial $F_{p q}(x)$, Amer. Math. Monthly 71 (1964) 769-770.

[3] L. Carlitz and J. M. Thomas, Rational tabulated values of trigonometric functions, Amer. Math. Monthly 69 (1962) 789-793.

[4] R. K. Guy, The strong law of small numbers, Amer. Math. Monthly 95 (1988) 697-712.

[5] T. W. Hungerford, Algebra, Springer-Verlag, New York, 1980.

[6] T. Y. Lam and K. H. Leung, On the cyclotomic polynomial $\Phi_{p q}(X)$, Amer. Math. Monthly 103 (1996) 562-564.

[7] D. H. Lehmer, A note on trigonometric algebraic numbers, Amer. Math. Monthly 40 (1933) 165-166.

[8] J. Suzuki, On coefficients of cyclotomic polynomials, Proc. Japan Acad. Ser. A Math. Sci. 63 (1987) 279-280.

Dr. Gregory Dresden (540) 458-8806 Department of Mathematics, Robinson Hall dresdeng@wlu .edu Washington and Lee University Lexington, VA 24450 